\title{
Emergency finger fractures - An easy fix
}

\author{
Michael Bell MD FRCSC FACS
}

M any finger fractures can be treated with low-technology open or closed pinning. With our ability to provide effective local anesthesia and hemostasis $(1,2)$, the only reason to transfer the patient from the emergency department to the main operating room is to utilize power equipment to drive $\mathrm{K}$ wires and $\mathrm{C}$-wires to obtain the desired reduction and fixation.

Many surgeons were quite comfortable using the Riordan pin, which has been taken off the market. Many surgeons have also used simple hypodermic needles, bending the tip slightly forward into the long axis of the needle so that it can be driven straight. Unfortunately, due to the hard bones of young male patients and the somewhat insecure attachment of the plastic needle to the plastic syringe, it can sometimes be difficult to efficiently pin some fractures.

$\mathrm{K}$-wires have an unsuitable tip configuration and need to be driven at high rotation and pressure into bone (with accompanying undesirable heat) (3). C-wires are a great mechanical improvement. They can be driven more slowly with higher torque. They cut efficiently and less traumatically into bone, and are reminiscent of the chairmaker's bits of the 19 th century.

I have devised a simple, modified vise grip as an effective pin driver, which I have used on hundreds of occasions in the emergency department (Figure 1). Using this tool, I have been able to fix virtually any fracture suitable for pinning without the significant inconvenience of obtaining time in the operating room.

Initially, the vise grip was used successfully without any modification. The grip was somewhat less precise and secure, so a slight modification (the creation of a slot) was devised. The slot was created with the use of a Dremel tool (Dremel, USA) and a fine cutting disk. A slot could be made equally simply with a fine triangular file, although likely with less accuracy and precision.

As seen in Figure 1, a simple longitudinal slot is cut into one jaw of the vise grip, angled very slightly off centre so that

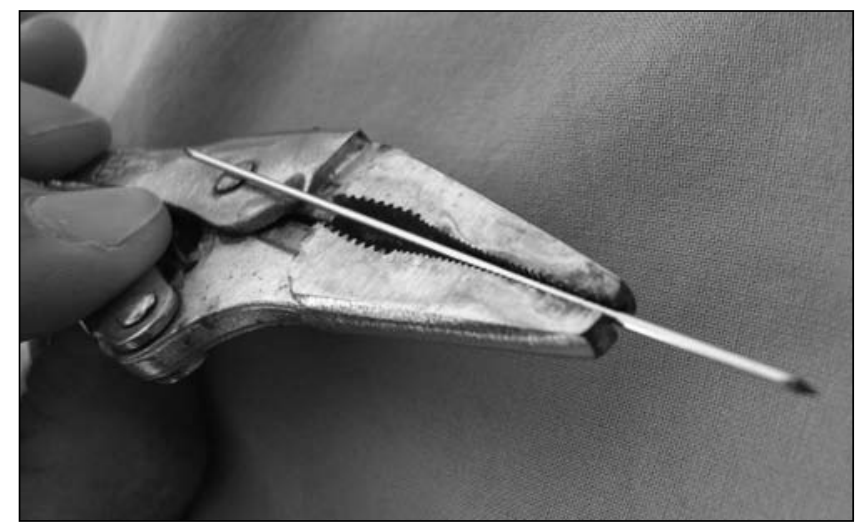

Figure 1) Needle driver created from a modified vise grip

the $\mathrm{C}$-wire rests without tension along the handle. The vise grip holds the pin absolutely securely, which can then be driven manually under controlled rotation and pressure into the bone and across the fracture.

The vise grip even has a built-in wire cutter, further minimizing the equipment required.

I have never had a complication using this simple device as described. It can be autoclaved numerous times before some slight rusting is noted. It can then, of course, be retired back to the workshop, where there are no cosmetic concerns, to continue its useful existence.

\section{REFERENCES}

1. Bell MSG, Reitsma BJ. Solo hand surgery. Can J Plast Surg 2005;13:145-7.

2. Guirguis E, Bell MS. The wrist tourniquet: An alternative technique in hand surgery. J Hand Surg (Am) 1990;15:516-9.

3. Ellabban MG, Oudit D, Juma A. Power drill radiator. Plast Reconstr Surg 2004;114:1970-1.

The Ottawa Hospital, Ottawa, Ontario

Correspondence and reprints: Dr Michael Bell, The Ottawa Hospital, 402-1919 Riverside Drive, Ottawa, Ontario K1H 1 A2.

Telephone 613-739-5424, fax 613-739-7168, e-mail msgbell@cyberus.ca 